\title{
Solvent and bio-degradable catalyst for the construction of fully-substituted benzenes
}

\author{
Ramesh Goud Koduri ${ }^{1}$, Ramakanth Pagadala ${ }^{1}$, Ravi Varala ${ }^{2}$ \\ ${ }^{1}$ Asst. Professor, CVR College of Engineering/H\&S Department (Chemistry), Hyderabad, India \\ Email: koduri.rameshgoud@gmail.com \\ ${ }^{1}$ Asst. Professor, CVR College of Engineering/H\&S Department (Chemistry), Hyderabad, India \\ Email: pagadalaramakanth@gmail.com \\ ${ }^{2}$ Professor, RGUKT Basar/Department of Chemistry, Niramal, India \\ Email: ravivarala@gmail.com
}

\begin{abstract}
An economical and efficient green chemical method is developed for the synthesis of multi-functionalized benzene derivatives through a natural biopolymer Cellulose Sulfuric Acid (CSA) as a heterogeneous catalytic sequence of Knoevenagal/Michael/Cyclization under solvent-free conditions at $70^{\circ} \mathrm{C}$. The metal-free catalytic system under solvent-free condition processes the reactions with a good efficiency. This protocol should be amenable advantages such as simple workup and recoverability of the biopolymer catalyst and reusing several times without significant decrease in catalytic activity.
\end{abstract}

Index Terms: Green synthesis, cellulose sulfuric acid, heterogeneous catalyst, multi-functionalized benzenes.

\section{INTRODUCTION}

Metal-free catalyst reactions promote more advantageous in chemical transformation since this catalytic reaction forms several bonds from easily available reactants and also reduces the isolated intermediates [1]. In addition, reactions in solvent-free conditions are recognized as unique selectivity, great implements for the synthesis of organic compounds and the products are readily formed in a single step [2].

Multicomponent reaction protocols involving domino processes, which combine multi reactants in a single step, can be powerfully accomplished by employing heterogeneous catalyst [3]. This approach has attracted considerable interest for the formation of multiple bonds that are formed in one-pot process and it addresses the basic principles of synthetic efficacy and reaction strategy arising from minimization of waste, time, energy, and cost [4].

In organic, natural products chemistry and material science Multi-functionalized benzenes holding 2, 6dicyanoaniline groups are essential class of compounds for asymmetric synthesis [5]. In olden days electrophilic and nucleophilic substitution on existed benzene ring [6], directed to metallation [7], metal-catalyzed cross-couplings of halo-aromatics or aryl triflates [8] were very commonly employed for the synthesis of multi-functionalized benzene derivatives. Various synthetic methods were described for the multi-functionalized benzene derivatives like the reaction between cycloalkylidenemalononitriles and arylmethylenecyanoacetamides in boiling ethanol catalyzed by piperidine [9], $\mathrm{NaOH}$ [10], $\mathrm{DBU}$ [11], $\mathrm{Et}_{3} \mathrm{~N}$ [12] and ethane diamine [13]. The reported synthetic protocols have more disadvantages like long reaction time, toxic reagents and prolonged reaction procedure. Moreover the catalysts were not easily regenerated and reused efficiently [14]. In this context it is desirable to develop a convenient and rapid method for the synthesis of multi functionalized benzene derivatives.

However, several research efforts have concentrated on the CSA as heterogeneous catalyst for the synthesis of $\alpha$ amino nitriles [15], aryl-dibenzo[a.j]xanthenes [16], 1,4dihydropyridines [17], Pechmann condensation [18], thiadiazolobenzimidazoles [19], imidazoazines [20], quinolines [21] and 3,4-dihydropyrimidine-2(1H)-ones [22]. These are evident from the literature that CSA supported catalyst has appeal to huge importance as a green catalyst to build carbon-carbon bonds in several organic transformations.

In this regards the synthesis of multi-functionalized benzene derivatives catalyzed by heterogeneous natural biopolymer cellulose sulfuric acid is more attractive [23]. This cellulose biopolymer catalyst is more abundant, biodegradable and renewable natural compounds in the world which has been in the center of attention over the past several decades [24]. The unique property of CSA is more attractive and replaced old organic and inorganic catalytic applications. The additional advantages of CSA are easy regeneration and efficient reuse.

\section{RESUlts AND Discussion}

Primarily, in search of the best solvent system for the synthesis of multi-functionalized benzenes, optimization of various reaction parameters like different temperature, amount of catalyst and solvents were carried out (Table 1). The reaction involves aromatic aldehyde, 2 equivalents of malononitrile and cycloheptanone in the presence of CSA under solvent-free conditions at $70^{\circ} \mathrm{C}$ (Scheme 1). The scope of this experiment was further demonstrated by studying the reaction of various aromatic aldehydes with malononitrile and cyclic ketone under described reaction conditions (Table 1 , entry 10) for the synthesis of multi-functionalized benzenes with good yields.

The comparative study of catalyst was done by performing the experiment with and without catalyst. The desired product was not observed by without catalyst (Table 1 ; entry 1, 2 and 8). The same reaction was performed in the 
presence of catalyst. Impressively, the metal-free catalyst method yielded the target compound selectively in $98 \%$ yield.

Reaction performed under various solvents like $\mathrm{EtOH}$, $\mathrm{H}_{2} \mathrm{O}$ and solvent free conditions and high conversion (98\%) was observed using $20.0 \mathrm{mg} \mathrm{CSA}$ in solvent-free at $70^{\circ} \mathrm{C}$ for $2 \mathrm{~h}$. The target compounds were not observed with water as solvent and CSA catalyst under different temperature conditions. The optimum reaction conditions obtained under solvent free conditions with $10.0 \mathrm{mg}, 20.0 \mathrm{mg}$ and $30.0 \mathrm{mg}$ of catalyst (CSA), in less reaction period of $2 \mathrm{~h}$ and at a temperature of $70^{\circ} \mathrm{C}$.

A suitable reaction mechanism for the construction of multi-substituted benzenes catalyzed by biodegradable CSA catalyst is shown in Scheme 2. The reaction was anticipated that CSA might preferentially form a Knoevenagel condensation product from aldehyde with malononitrile and followed by Mannich-type reaction with cycloheptanone. Finally, the HCN molecule is eliminated by attacking of another Cyanoacetonitrile molecule.

Heterogeneous catalysis is more attractive due to its profound advantages of straightforward reusable characteristic. The CSA catalyst was recycled and observed through the synthesis of $4 \mathrm{a}$. The crude reaction mixture was dissolved in ethyl acetate after completion of the reaction. It was filtered and then recovered; the recovered catalyst can be reused for five times without loss of its efficiency (Table 2 ). Due to these positive results, all the reactions were supported out under identical conditions.

The scope of this method was further developed by studying the reaction of various substrates (Table 3 ) in the presence of $20 \mathrm{mg}$ catalyst in similar reaction conditions, the desired compounds were obtained with good yield. It was found that the substituted aromatic aldehydes with both electron-withdrawing and donating groups, in reaction with other easily available starting materials, had good isolated yields.

\section{EXPERIMENTAL}

\section{Preparation of Cellulosic Sulfuric acid}

Take cellulose (5.00 gm), add n-hexane $(20 \mathrm{ml})$ and magnetically stirred for some period. Then $9 \mathrm{mmol}$ of chlorosulfonic acid $(1.0 \mathrm{~g})$ was added drop by drop for $2 \mathrm{~h}$ at $0^{\circ} \mathrm{C}$ and remove $\mathrm{HCl}$ from the vessel. The mixture was then stirred for about $2.5 \mathrm{~h}$ at $25^{\circ} \mathrm{C}$. The filtered mixture was washed with acetonitrile and dried completely at room temperature. The white color cellulosic sulfuric acid powder was weighed and noted as $5.24 \mathrm{~g}$.

Process for the synthesis of multi-functionalized benzene

A mixture of benzaldehyde (1 mmol), malononitrile (2 mmol), cycloheptanone (1 mmol) and $20.0 \mathrm{mg}$ of CSA added in sequence, stirred magnetically at $70^{\circ} \mathrm{C}$ for appropriate time shown in Table 1. The reaction progress was checked by TLC $($ Hexane/EtOAc $=4: 1)$. The reaction mixture was allowed to room temperature and added ethyl acetate after completion of the reaction. The obtained solid was filtered, washed with ethyl ethanoate, dried with anhydrous $\mathrm{Na}_{2} \mathrm{SO}_{4}$ and the solvent was evaporated. The attained precipitate was re-crystallized from ethyl alcohol to get the pure off white solid objective compound 4a (98\% yield). The recollected catalyst CSA was washed with chloroform, solvent evaporated and the same catalyst reused for the next cycle.

4-phenyl-2-amino-6, 7,8,9-tetrahydro-5H-benzo[7] annulene1,3-dicarbonitrile $(4 a)$

Off white solid: mp $217-218^{\circ} \mathrm{C}$; ${ }^{13} \mathrm{C}$ NUCLEAR MAGNETIC RESONANCE SPECTRA $\delta$ 26.92, 27.58, $30.27,31.57,34.82$, 96.06, 97.26, 115.84, 115.87, 128.43, $128.66,128.92,132.25,137.77,148.75,149.79,153.53 ;{ }^{1} \mathrm{H}$ NUCLEAR MAGNETIC RESONANCE SPECTRA $\delta=$ $1.42-1.83(6 \mathrm{H}, \mathrm{m}), 2.46(2 \mathrm{H}, \mathrm{t}, \mathrm{J}=5.4 \mathrm{~Hz}), 3.08(2 \mathrm{H}, \mathrm{t}, \mathrm{J}=$ $5.2 \mathrm{~Hz}), 5.04\left(2 \mathrm{H}, \mathrm{s}, \mathrm{NH}_{2}\right), 7.19-7.50(5 \mathrm{H}, \mathrm{m})$; Mass spectra, $\mathrm{m} / \mathrm{z}=288$ Analytical calculation $\left(\mathrm{C}_{19} \mathrm{H}_{17} \mathrm{~N}_{3}\right): \mathrm{N} 14.62 \%, \mathrm{C}$ $79.41 \%$, H 5.96\%. Found: N 14.63\%, C 79.49\%, H 5.98\%; INFRA RED SPECTRA $3345\left(\mathrm{NH}_{2}\right), 2221(\mathrm{CN})$.

4-(4-methoxyphenyl)- 2-amino-6, 7,8,9-tetrahydro-5Hbenzo[7] annulene-1,3-dicarbonitrile (4b)

Off white solid: mp $199-200{ }^{\circ} \mathrm{C} ; \quad{ }^{13} \mathrm{C}$ NUCLEAR MAGNETIC RESONANCE SPECTRA $\delta$ 26.93, 27.63, $30.23,31.59,34.82,55.27,96.36,97.03,114.07,115.90$, $116.08,129.82,129.92,132.61,148.64,149.76,153.41$, 159.78; ${ }^{1} \mathrm{H}$ NUCLEAR MAGNETIC RESONANCE SPECTRA $\delta=1.44-1.79(6 \mathrm{H}, \mathrm{m}), 2.51(2 \mathrm{H}, \mathrm{t}, \mathrm{J}=5.3 \mathrm{~Hz})$, $3.05(2 \mathrm{H}, \mathrm{t}, \mathrm{J}=5.1 \mathrm{~Hz}), 3.87\left(3 \mathrm{H}, \mathrm{s},-\mathrm{OCH}_{3}\right), 5.02(2 \mathrm{H}, \mathrm{s}$, $\left.\mathrm{NH}_{2}\right), 6.98(2 \mathrm{H}, \mathrm{d}, \mathrm{J}=8.6), 7.11(2 \mathrm{H}, \mathrm{d}, \mathrm{J}=8.6)$; Mass spectra, $\mathrm{m} / \mathrm{z}=318$ Analytical calculation $\left(\mathrm{C}_{20} \mathrm{H}_{19} \mathrm{~N}_{3} \mathrm{O}\right): \mathrm{N}$ $13.24 \%$, C 75.69\%, H 6.03\%. Found: N 13.25\%, C $75.77 \%$, H 6.08\%; INFRA RED SPECTRA $3346\left(\mathrm{NH}_{2}\right)$, $2217(\mathrm{CN})$.

4-(4-bromophenyl)- 2-amino-6, 7,8,9-tetrahydro-5Hbenzo[7] annulene-1,3-dicarbonitrile (4c)

Pale yellow solid: mp $228-229^{\circ} \mathrm{C} ;{ }^{13} \mathrm{C}$ NUCLEAR MAGNETIC RESONANCE SPECTRA $\delta$ 26.89, 27.56, $30.28,31.52$, 34.83, 95.66, 97.62, 115.69, 115.73, 123.12, $130.18,131.98,132.14,136.61,147.33,149.85,153.79 ;{ }^{1} \mathrm{H}$ NUCLEAR MAGNETIC RESONANCE SPECTRA $\delta=$ 1.45-1.79 (6H, m), $2.44(2 \mathrm{H}, \mathrm{t}, \mathrm{J}=5.4 \mathrm{~Hz}), 3.06(2 \mathrm{H}, \mathrm{t}, \mathrm{J}=$ $5.3 \mathrm{~Hz}), 5.09\left(2 \mathrm{H}, \mathrm{s}, \mathrm{NH}_{2}\right), 7.06(2 \mathrm{H}, \mathrm{d}, \mathrm{J}=8.4), 7.59(2 \mathrm{H}$, $\mathrm{d}, \mathrm{J}=8.3$ ); Mass spectra, $\mathrm{m} / \mathrm{z}=388$ ANALYTICAL CALCULATION $\left(\mathrm{C}_{19} \mathrm{H}_{16} \mathrm{BrN}_{3}\right)$ : N $11.47 \%$, C 62.31\%, H $4.40 \%$. Found: N $11.56 \%$, C $62.26 \%, \mathrm{H} 4.45 \%$; INFRA RED SPECTRA $3347\left(\mathrm{NH}_{2}\right), 2218(\mathrm{CN})$.

4-(4-hydroxyphenyl)- 2-amino-6, 7,8,9-tetrahydro-5Hbenzo[7] annulene-1,3-dicarbonitrile (4d)

Off white solid: $\mathrm{mp} \quad 240-241^{\circ} \mathrm{C}$; ${ }^{13} \mathrm{C}$ NUCLEAR MAGNETIC RESONANCE SPECTRA $\delta$ 26.93, 27.62, 30.22, 31.58, 34.83, 96.27, 97.10, 115.62, 115.87, 116.09, $130.02,131.98,132.14,148.57,149.76,153.49,156.01 ;{ }^{1} \mathrm{H}$ NUCLEAR MAGNETIC RESONANCE SPECTRA $\delta=$ 1.44-1.79 (6H, m), $2.51(2 \mathrm{H}, \mathrm{t}, \mathrm{J}=5.4 \mathrm{~Hz}), 3.05(2 \mathrm{H}, \mathrm{t}, \mathrm{J}=$ $5.3 \mathrm{~Hz}), 5.02\left(2 \mathrm{H}, \mathrm{s}, \mathrm{NH}_{2}\right), 5.24(1 \mathrm{H}, \mathrm{s},-\mathrm{OH}), 6.89(2 \mathrm{H}, \mathrm{d}, \mathrm{J}$ $=8.4), 7.06(2 \mathrm{H}, \mathrm{d}, \mathrm{J}=8.4) ;$ Mass spectra, $\mathrm{m} / \mathrm{z}=326$ ANALYTICAL CALCULATION $\left(\mathrm{C}_{17} \mathrm{H}_{17} \mathrm{~N}_{3} \mathrm{O}\right)$ : N $13.85 \%$, C $75.23 \%$, H 5.65\%. Found: N 13.83\%, C 75.30\%, H 5.68\%; INFRA RED SPECTRA $3342\left(\mathrm{NH}_{2}\right), 2204(\mathrm{CN})$. 
4-(4-(dimethylamino)phenyl)- 2-amino-6,7,8,9-tetrahydro5H-benzo[7] annulene-1,3-dicarbonitrile (4e)

Pale yellow solid: mp $229-230^{\circ} \mathrm{C} ;{ }^{13} \mathrm{C}$ NUCLEAR MAGNETIC RESONANCE SPECTRA $\delta$ 26.98, 27.74, $30.20,31.66,34.81,40.24,96.48,96.50,111.80,116.14$, $116.47,127.65,129.60,132.71,149.55,149.84,150.34$, 153.14; ${ }^{1} \mathrm{H}$ NUCLEAR MAGNETIC RESONANCE SPECTRA $\delta=1.47-1.79(6 \mathrm{H}, \mathrm{m}), 2.55(2 \mathrm{H}, \mathrm{t}, \mathrm{J}=5.4 \mathrm{~Hz})$, $3.02\left(6 \mathrm{H}, \mathrm{s},\left(-\mathrm{NCH}_{3}\right)_{2}\right), 3.06(2 \mathrm{H}, \mathrm{t}, \mathrm{J}=5.3 \mathrm{~Hz}), 5.1(2 \mathrm{H}, \mathrm{s}$, $\left.\mathrm{NH}_{2}\right), 6.74(2 \mathrm{H}, \mathrm{d}, \mathrm{J}=8.7), 7.05(2 \mathrm{H}, \mathrm{d}, \mathrm{J}=8.7)$; Mass spectra, $\mathrm{m} / \mathrm{z}=331$ ANALYTICAL CALCULATION $\left(\mathrm{C}_{21} \mathrm{H}_{22} \mathrm{~N}_{4}\right): \mathrm{N} 16.96 \%$, C 76.33\%, H 6.71\%. Found: $\mathrm{N}$ $16.99 \%$, C 76.39\%, H 6.78\%; INFRA RED SPECTRA 3341 $\left(\mathrm{NH}_{2}\right), 2211(\mathrm{CN})$.

4-(2-chlorophenyl)- 2-amino-6, 7,8,9-tetrahydro-5Hbenzo[7] annulene-1,3-dicarbonitrile (4f)

Off white solid: mp $236-237^{\circ} \mathrm{C} ;{ }^{13} \mathrm{C}$ NUCLEAR MAGNETIC RESONANCE SPECTRA $\delta$ 26.89, 27.17, $30.53,31.56,34.94,95.83,97.96,115.32,115.74,127.22$, $129.93,130.07,130.32,132.51,132.82,136.65,145.72$, 149.84, 153.67; ${ }^{1} \mathrm{H}$ NUCLEAR MAGNETIC RESONANCE SPECTRA $\delta=1.36-1.78(6 \mathrm{H}, \mathrm{m}), 2.37-2.43(2 \mathrm{H}, \mathrm{m}), 3.08$ $(2 \mathrm{H}, \mathrm{t}, \mathrm{J}=4.9 \mathrm{~Hz}), 5.09\left(2 \mathrm{H}, \mathrm{s}, \mathrm{NH}_{2}\right), 7.14-7.53(4 \mathrm{H}, \mathrm{m})$; Mass spectra, $\mathrm{m} / \mathrm{z}=322$ ANALYTICAL CALCULATION $\left(\mathrm{C}_{19} \mathrm{H}_{16} \mathrm{ClN}_{3}\right): \mathrm{N} 13.06 \%$, C 70.91\%, H 5.01\%. Found: $\mathrm{N}$ $13.11 \%$, C 70.98\%, H 5.06\%; INFRA RED SPECTRA 3245 $\left(\mathrm{NH}_{2}\right), 2223(\mathrm{CN})$. 4-(2-bromophenyl)- 2-amino-6, 7,8,9-tetrahydro-5Hbenzo[7]annulene-1,3-dicarbonitrile (4g)

Light yellow solid: mp $256-257^{\circ} \mathrm{C}$; ${ }^{13} \mathrm{C}$ NUCLEAR MAGNETIC RESONANCE SPECTRA $\delta$ 26.87, 27.10, $30.51,31.57,34.91,95.78,97.96,115.32,115.72,122.66$, $127.79,129.96,130.38,132.29,133.06,138.72,147.30$, 149.83, 153.75; ${ }^{1} \mathrm{H}$ NUCLEAR MAGNETIC RESONANCE SPECTRA $\delta=1.37-1.79(6 \mathrm{H}, \mathrm{m}), 2.32-2.45(2 \mathrm{H}, \mathrm{m}), 3.07$ $(2 \mathrm{H}, \mathrm{t}, \mathrm{J}=4.8 \mathrm{~Hz}), 5.09\left(2 \mathrm{H}, \mathrm{s}, \mathrm{NH}_{2}\right), 7.14-7.72(4 \mathrm{H}, \mathrm{m})$; Mass spectra, $\mathrm{m} / \mathrm{z}=366$ ANALYTICAL CALCULATION $\left(\mathrm{C}_{19} \mathrm{H}_{16} \mathrm{BrN}_{3}\right): \mathrm{N} 11.47 \%$, C $62.31 \%, \mathrm{H} 4.40 \%$. Found: $\mathrm{N}$ $11.53 \%$, C $62.35 \%$, H 4.46\%; INFRA RED SPECTRA 3342 $\left(\mathrm{NH}_{2}\right), 2225(\mathrm{CN})$.

4-(2-methoxyphenyl)- 2-amino-6, 7,8,9-tetrahydro-5Hbenzo[7] annulene-1,3-dicarbonitrile (4h)

Off white solid: $\mathrm{mp} \quad 210-211^{\circ} \mathrm{C} ;{ }^{13} \mathrm{C}$ NUCLEAR MAGNETIC RESONANCE SPECTRA $\delta$ 26.93, 27.25, $30.61,31.73,34.89$, 55.45, 96.61, 97.35, 111.10, 115.99, $120.78,126.45,130.06,130.43,133.11,145.81,149.77$, 153.04, 156.21; ${ }^{1} \mathrm{H}$ NUCLEAR MAGNETIC RESONANCE SPECTRA $\delta=1.42-1.76(6 \mathrm{H}, \mathrm{m}), 2.42(2 \mathrm{H}, \mathrm{t}, \mathrm{J}=5.2 \mathrm{~Hz})$, $3.05(2 \mathrm{H}, \mathrm{t}, \mathrm{J}=5.3 \mathrm{~Hz}), 3.78\left(3 \mathrm{H}, \mathrm{s},-\mathrm{OCH}_{3}\right), 4.9(2 \mathrm{H}, \mathrm{s}$, $\mathrm{NH}_{2}$ ), 6.98-7.43 (4H, m); Mass spectra, $\mathrm{m} / \mathrm{z}=318$ ANALYTICAL CALCULATION $\left(\mathrm{C}_{20} \mathrm{H}_{19} \mathrm{~N}_{3} \mathrm{O}\right)$ : N $13.24 \%$, C $75.69 \%, \mathrm{H} \quad 6.03 \%$, Found: $\mathrm{N} 13.27 \%, \mathrm{C} 75.75 \%, \mathrm{H}$ 6.04\%; INFRA RED SPECTRA $3350\left(\mathrm{NH}_{2}\right), 2219(\mathrm{CN})$.

TABLE I.

Optimized REACTION CONDITIONS For THE SYNTHESIS OF FUlly SUBSTITUTED BENZENE DERIVATIVES

\begin{tabular}{|c|c|c|c|c|c|c|c|}
\hline Entry & Compound & Catalyst & Quantity & Solvent & Temperature $\left({ }^{\circ} \mathrm{C}\right)$ & Time (h) & Yield ${ }^{\mathrm{a}}(\%)$ \\
\hline 1 & $4 a$ & - & - & $\mathrm{EtOH}$ & Room temperature & 12.0 & $\mathrm{~b}$ \\
\hline 2 & $4 a$ & - & - & $\mathrm{EtOH}$ & 70 & 12.0 & $\mathrm{~b}$ \\
\hline 3 & $4 a$ & CSA & $10.0 \mathrm{mg}$ & $\mathrm{EtOH}$ & Room temperature & 6.0 & 40 \\
\hline 4 & $4 a$ & CSA & $10.0 \mathrm{mg}$ & $\mathrm{EtOH}$ & 60 & 5.0 & 56 \\
\hline 5 & $4 a$ & CSA & $10.0 \mathrm{mg}$ & $\mathrm{H}_{2} \mathrm{O}$ & Room temperature & 6.0 & $\mathrm{~b}$ \\
\hline 6 & $4 a$ & CSA & $10.0 \mathrm{mg}$ & $\mathrm{H}_{2} \mathrm{O}$ & 80 & 5.0 & b \\
\hline 7 & $4 a$ & CSA & $10.0 \mathrm{mg}$ & - & Room temperature & 2.5 & 82 \\
\hline 8 & $4 a$ & - & - & - & 70 & 4.0 & b \\
\hline 9 & $4 a$ & CSA & $10.0 \mathrm{mg}$ & - & 70 & 2.0 & 94 \\
\hline 10 & $4 a$ & CSA & $20.0 \mathrm{mg}$ & - & 70 & 2.0 & 98 \\
\hline 11 & $4 a$ & CSA & $30.0 \mathrm{mg}$ & - & 70 & 2.0 & 98 \\
\hline
\end{tabular}

${ }^{a}$ Isolated yields

${ }^{b}$ No products found.

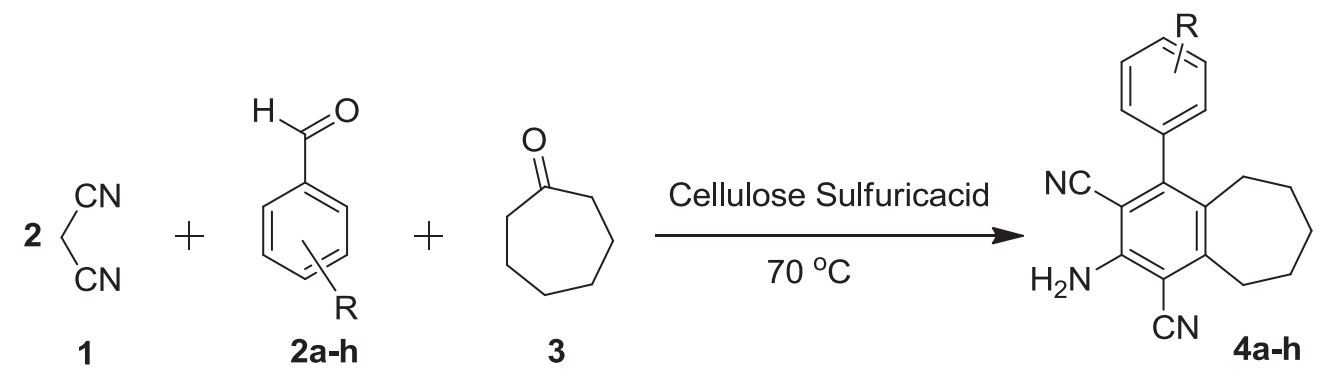

Scheme 1. Cyclo-condnsation reaction for the synthesis of fully-substituted benzenes (4a-h) 


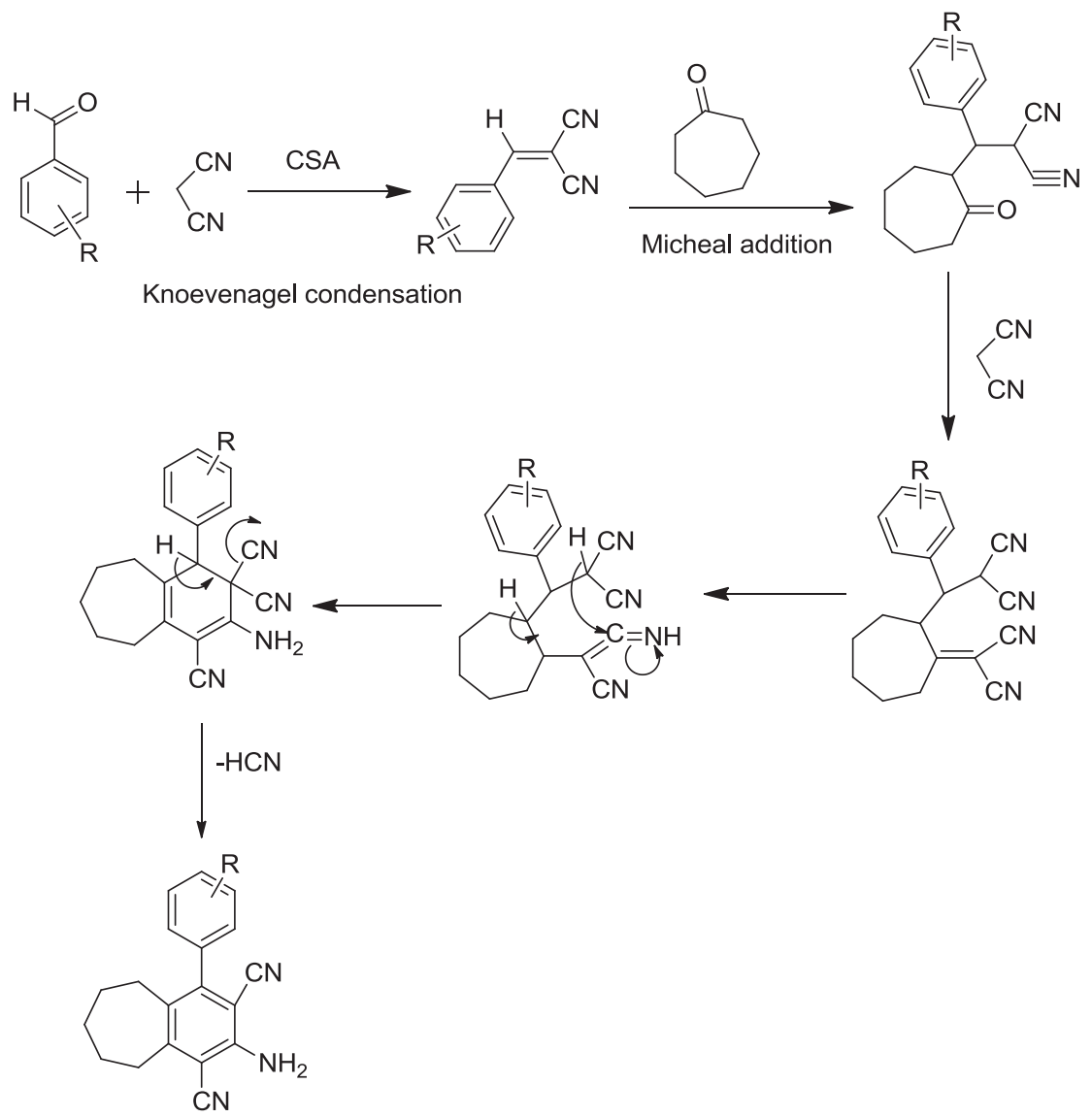

Scheme 2. A plausible reaction mechanism for the formation of fully-substituted benzenes

TABLE II.

ReusABILITY OF CSA CATALYST CONFIRMED For COMPOUND (4a)

\begin{tabular}{cccc}
\hline Entry & Catalyst & $\begin{array}{c}\text { Time } \\
(\mathrm{h})\end{array}$ & $\begin{array}{c}\text { Yield } \\
\mathrm{a}(\%)\end{array}$ \\
\hline 1 & Fresh & 2.0 & 98.0 \\
2 & $2^{\text {nd }}$ run & 2.0 & 98.0 \\
3 & $3^{\text {rd }}$ run & 2.0 & 97.0 \\
4 & $4^{\text {th }}$ run & 2.0 & 95.0 \\
5 & $5^{\text {th }}$ run & 2.0 & 94.0
\end{tabular}

TABLE III.

Csa Catalyzed Synthesis Of The Fully-Substituted BenzenE DERIVATIVES (4a-h)

\begin{tabular}{ccccc}
\hline Entry & $\begin{array}{c}\text { Product } \\
\text { No. }\end{array}$ & $\mathrm{R}_{1}$ & Time (h) & $\begin{array}{c}\text { Yield } \\
\mathrm{a}(\%)\end{array}$ \\
\hline 1 & $4 \mathrm{a}$ & $\mathrm{H}$ & 2.0 & 98.0 \\
2 & $4 \mathrm{~b}$ & $4-\mathrm{OCH}_{3}$ & 2.5 & 98.0 \\
3 & $4 \mathrm{c}$ & $4-\mathrm{Br}$ & 2.5 & 97.0 \\
4 & $4 \mathrm{~d}$ & $4-\mathrm{OH}$ & 2.5 & 96.0 \\
5 & $4 \mathrm{e}$ & $4-\mathrm{N}\left(\mathrm{CH}_{3}\right)_{2}$ & 2.0 & 95.0 \\
6 & $4 \mathrm{f}$ & $2-\mathrm{Cl}$ & 2.0 & 97.0 \\
7 & $4 \mathrm{~g}$ & $2-\mathrm{Br}$ & 2.0 & 96.0 \\
8 & $4 \mathrm{~h}$ & $2-\mathrm{OCH} \mathrm{H}_{3}$ & 2.5 & 95.0 \\
\hline
\end{tabular}

\section{CONCLUSIONS}

In conclusion cellulose sulfuric acid was applied in simple and straightforward method for the efficient synthesis of various multi-functionalized benzene derivatives. CSA is an effective, harmless, ecological and environmental friendly solid acid catalyst. This protocol has many advantages such as scale-up, simplicity of experimental process, easy work-up, high yields and green conditions. So far great advances have been developed in research the usage of new CSA catalyst in organic synthesis of pharmaceutical curiosity is obligatory. Further studies in the sustainable applications of this biopolymer catalyst to other organic chemical reactions are continuing in our laboratory.

\section{REFERENCES}

[1] (a) K. C. Nicolaou, D. J. Edmonds and P. G. Bulger, Angew. Chem., Int. Ed., 2006, 45, 7134; (b) J. C. Wasilke, S. J. Obrey, R. T. Baker and G. C. Bazan, Chem. Rev., 2005, 105, 1001.

[2] A. Domling and I. Ugi, Angew. Chem., Int. Ed., 2000, 39, 3168.

[3] (a) R. Pagadala, S. Maddila, V. Moodley, W. E. Van Zyl, S. B. Jonnalagadda, Tetrahedron Lett., 2014, 55, 4006; (b) R. Pagadala, S. Maddila, S. Rana, S. B. Jonnalagadda, RSC Adv., 2014, 4, 6602; (c) U. Kusampally, R. Pagadala, K. C. Rajanna; Tetrahedron Letters 2017, 58, 3316. 
[4] N. G. Shabalala, R. Pagadala, S. B. Jonnalagadda; Ultrasonics Sonochemistry, 2015, 27, 423; (b) R. Pagadala, D. R. Kommidi, S. Kankala, S. Maddila, P. Singh, B. Moodley, N. A. Koorbanally, S. B. Jonnalagadda; Organic and Biomolecular Chemistry 2015, 13, 1800; (c) R. Pagadala, S. Maddila, S. B. Jonnalagadda; Journal of Heterocyclic Chemistry 2015, 52, 1226.

[5] (a) X. S. Wang, M. M. Wang, Q. Li, C. S. Yao, S. J. Tu, Tetrahedron, 2007, 63, 5265; (b) F. V. Singh, R. Vatsyayan, U. Roy, A. Goel, Bioorg. Med. Chem. Lett., 2006, 16, 2734; (c) F. Dumur, N. Gautier, N. Gallego-Planas, Y. Sahin, E. Levillain, N. Mercier, P. Hudhomme, J. Org. Chem., 2004, 69, 2164; (d) Y. Xiao, X. H. Qian, Tetrahedron Lett., 2003, 44, 2087; (e) N. J. Long, Angew. Chem. Int. Ed. Engl., 1995, 34, 21; (f) N. G. Andersen, S. P. Maddaford, B. A. Keay, J. Org. Chem., 1996, 61, 9556; (g) H. Kurreck, M. Huber, Angew. Chem. Int. Ed. Engl., 1995, 34, 849.

[6] (a) R. Taylor, Electrophilic Aromatic Substitution; Wiley: Chichester, UK, 1990; (b) J. Miller, Aromatic Nucleophilic Substitution; Elsevier: Amsterdam, 1968.

[7] V. Snieckus, Chem. Rev. 1990, 90, 879.

[8] de Meijere, A.; Diederich, F. Metal-Catalyzed Cross-Coupling reactions; Wiley: Weinheim, 2004.

[9] (a) G. E. H. Elgemeie and F. A. Abdelaal, Heterocycles, 1986, 24, 349. (b) G. E. H. Elgemeie and M. M. Ramiz, Phosphorus Sulfur, 1989, 46, 95; (c) G. E. H. Elgemeie, H. A. Elfahham and H. Nabey, Sulfur Lett., 1989, 9; (d) A. C. Cope and K. E. Hoyle, J. Org. Chem., 1941, 63, 733.

[10] (a) L. Rong, H. Han, H. Jiang, S. Tu, Synth. Commun., 2008, 38, 3530; (b) H. Han, F. Yang, H. Yao, Synth. Commun., 2007, 37, 3767.

[11] R. Liangce, T. Shimin, X. Sheng, L. Lihua, Y. Shan,S. Yanhui, Res. Chem. Intermed., 2012, 38, 1647.

[12] S. L. Cui, X. F. Lin, Y. G. Wang, J. Org. Chem., 2005, 70, 2866.

[13] J. Wang, Q. Li, C. Qi, Y. Liu, Z. Ge,R. Li, Org. Biomol. Chem., 2010, 8, 4240.

[14] (a) V. Raghukumar, P. Murugan, V. T. Ramakrishnan, Synth. Commun., 2001, 31, 3497; (b) P. J. Victory, J. I. Borrell, A. Vidal-Ferran, Heterocycles, 1993, 36, 769; (c) P. Victory, J. I. Borrel, A. Vidal-Ferran, C. Seoane, J. L. Soto, Tetrahedron Lett., 1991, 32, 5375.

[15] B. Leroy, I. E. Markó, Tetrahedron Lett., 2001, 42, 8685.

[16] S. Vidal, Synlett., 2001, 1194.

[17] M. D. Carrigan, D. Sarapa, R. C. Smith, L. C. Wieland, R. S. Mohan, J. Org. Chem., 2002, 67, 1027.

[18] M. V. Reddy, S. D. Dindulkar, Y. T. Jeong, Tetrahedron Lett., 2011, 52, 4764.

[19] S. Antoniotti, Synlett., 2003, 1566.

[20] R. Ramachandran, S. Jayanthi, Y. T. Jeong, Tetrahedron., $2012,68,363$.

[21] M. D. Carrigan, K. J. Eash, M. C. Oswald, R. S. Mohan, Tetrahedron Lett., 2001, 42, 8133.

[22] L. Wu, W. Ma, L. Yang, F. Yan, Asian J. Chem., 2010, 22, 6053.

[23] R. Breslow, Acc. Chem. Res., 1980, 13, 170.

[24] D. Klemm, B. Heublein, H. P. Fink, A. Bohn, Angew. Chem., Int. Ed., 2005, 44, 3358. 